\title{
Effects of long-term balance training with vibrotactile sensory augmentation among community-dwelling healthy older adults: a randomized preliminary study
}

Tian Bao ${ }^{1}$, Wendy J. Carender ${ }^{2}$, Catherine Kinnaird', Vincent J. Barone ${ }^{1}$, Geeta Peethambaran ${ }^{3}$, Susan L. Whitney ${ }^{4}$, Mohammed Kabeto ${ }^{5}$, Rachael D. Seidler ${ }^{6,7,8}$ and Kathleen H. Sienko ${ }^{1,9^{*}}$

\begin{abstract}
Background: Sensory augmentation has been shown to improve postural stability during real-time balance applications. Limited long-term controlled studies have examined retention of balance improvements in healthy older adults after training with sensory augmentation has ceased. This pilot study aimed to assess the efficacy of long-term balance training with and without sensory augmentation among community-dwelling healthy older adults.

Methods: Twelve participants (four males, eight females; $75.6 \pm 4.9 \mathrm{yrs}$ ) were randomly assigned to the experimental group $(n=6)$ or control group $(n=6)$. Participants trained in their homes for eight weeks, completing three 45 -min exercise sessions per week using smart phone balance trainers that provided written, graphic, and video guidance, and monitored trunk sway. During each session, participants performed six repetitions of six exercises selected from five categories (static standing, compliant surface standing, weight shifting, modified center of gravity, and gait). The experimental group received vibrotactile sensory augmentation for four of the six repetitions per exercise via the smart phone balance trainers, while the control group performed exercises without sensory augmentation. The smart phone balance trainers sent exercise performance data to a physical therapist, who recommended exercises on a weekly basis. Balance performance was assessed using a battery of clinical balance tests (Activity Balance Confidence Scale, Sensory Organization Test, Mini Balance Evaluation Systems Test, Five Times Sit to Stand Test, Four Square Step Test, Functional Reach Test, Gait Speed Test, Timed Up and Go, and Timed Up and Go with Cognitive Task) before training, after four weeks of training, and after eight weeks of training.

(Continued on next page)
\end{abstract}

\footnotetext{
* Correspondence: sienko@umich.edu

'Department of Mechanical Engineering, University of Michigan, 2350 Hayward St., Ann Arbor 48109, MI, USA

${ }^{9}$ Department of Biomedical Engineering, University of Michigan, 2200

Bonisteel Blvd, Ann Arbor, MI 48109, USA

Full list of author information is available at the end of the article
}

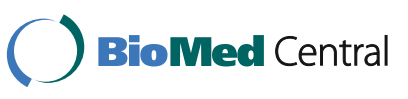

(c) The Author(s). 2018 Open Access This article is distributed under the terms of the Creative Commons Attribution 4.0 International License (http://creativecommons.org/licenses/by/4.0/), which permits unrestricted use, distribution, and reproduction in any medium, provided you give appropriate credit to the original author(s) and the source, provide a link to the Creative Commons license, and indicate if changes were made. The Creative Commons Public Domain Dedication waiver (http://creativecommons.org/publicdomain/zero/1.0/) applies to the data made available in this article, unless otherwise stated. 
(Continued from previous page)

Results: Participants in the experimental group were able to use vibrotactile sensory augmentation independently in their homes. After training, the experimental group had significantly greater improvements in Sensory Organization Test and Mini Balance Evaluation Systems Test scores than the control group. Significant improvement was also observed for Five Times Sit to Stand Test duration within the experimental group, but not in the control group. No significant improvements between the two groups were observed in the remaining clinical outcome measures.

Conclusion: The findings of this study support the use of sensory augmentation devices by community-dwelling healthy older adults as balance rehabilitation tools, and indicate feasibility of telerehabilitation therapy with reduced input from clinicians.

Keywords: Balance rehabilitation, Vibrotactile, Biofeedback, Sensory augmentation, Long-term, Older adults, Home-based, Smartphone balance trainer, Telerehabilitation, Wearable devices

\section{Background}

Age-related deterioration of sensory function, inefficient integration of sensory systems, and reduced muscle strength contribute to decreased balance performance in older adults [1-3]. Degradation of balance performance increases fall risk [4-6] and fear of falling [7], and inhibits mobility, thereby reducing independence and quality of life $[8,9]$.

Exercise-based rehabilitation programs are effective for improving balance performance in community-dwelling older adults [10]. Typical regimens emphasize building strength and stamina to counteract musculoskeletal degeneration, and balance training to isolate and challenge the somatosensory, visual, and vestibular systems [10, 11]. In particular, balance training leverages the ability of the central nervous system to "reweight" functioning sensory inputs [12]. Clinic-, group-, and home-based balance training programs guided by clinicians and lasting four to twelve weeks have yielded significant improvements in clinical outcome measures (e.g., Sensory Organization Test, Timed Up-and-Go) corresponding directly to reductions in fall rate, occurrence, and risk, and maintenance of social and physical activity [13-16]. Training programs without direct clinical guidance, however, are less effective $[17,18]$. Kao et al. reported that more participants in a supervised exercise program group showed clinically significant improvements than those performing the same exercises at home without supervision [17]. Lacroix et al. showed that a 12-week supervised balance and strength training program improved static, dynamic, proactive, and reactive measures of balance more than an unsupervised program in healthy older adults [18]. These studies suggest that monitoring performance and providing feedback during balance and strength training may improve program efficacy.

Sensory augmentation (SA) provides additional information to complement and/or replace native sensory input from the somatosensory, visual, and/or vestibular systems [19]. SA systems for balance applications typically employ one sensor or a network of sensors (e.g., motion capture, force plate, inertial measurement unit, goniometer) to measure body motion, and a display to communicate body motion and/or provide instructional cues. Most studies of balance-related SA have explored real-time usage applications as an assistive device in lieu of the somatosensory contact cues provided by a cane or walker to the fingertips [19-34]. During real-time use, it is hypothesized that the central nervous system incorporates SA as an additive input, supplementing other sensory information $[22,35,36]$. In individuals with balance deficits, healthy young adults, and healthy older adults, various SA modalities (e.g., vibrotactile [23-29], visual [30], auditory [31,32], electrotactile [19, 37], and multimodal [33]) have been shown to improve real-time balance performance by reducing body sway during static, dynamic, and perturbed standing tasks. While use of SA based on trunk sway during gait tasks (e.g., tandem walk, straight walk) has been shown to reduce trunk sway, the effects are limited [32, 38-40].

In addition to real-time assistive device applications, SA may be useful as a rehabilitation tool during traditional balance training. It is hypothesized that cues from SA may facilitate the central nervous system in "reweighting" sensory inputs during training to improve balance ability [22, 41]. To evaluate whether SA devices can be used as rehabilitation tools, it is important to analyze whether balance improvements observed during training persist after training is completed and SA is no longer provided.

Prior studies have shown that post-training improvements persist hours to days following short-term (i.e., less than one week) training with SA [23, 33, 42] and on the order of weeks to months following multi-session (i.e., more than one week) training with SA for people with balance deficits [43-45]. Rossi-Izquierdo et al. showed that after two weeks of exercise training with vibrotactile SA, people with Parkinson's disease reduced 
trunk sway on trained exercises and demonstrated improved clinical outcome measures (e.g., Dizziness Handicap Inventory, Sensory Organization Test) [43]. Furthermore, these improvements persisted three months after training [43]. Basta et al. reported similar improvements in a group of people with various balance disorders trained with vibrotactile SA, but found no such effect in a group trained with erroneous SA signals [44]. Brugnera et al. found improved clinical balance measures (e.g., Sensory Organization Test, Activities-specific Balance Confidence) among people with vestibular disorders following two weeks of balance training with vibrotactile SA, but found no improvements among participants trained following standard rehabilitation practices [45].

Limited studies have examined balance improvements after longer-term training with SA among healthy older adults. Video game-based in-home balance training was shown to improve clinical measures (e.g., maximal muscle strength, Activity-specific Balance Confidence, risk of falling) after a minimum of five weeks of training [46-49]. However, video game-based balance training typically requires a balance platform (e.g., Wii Fit balance board) and a display screen to provide visual cues, which can limit its utility during balance exercises that require closed eyes, head movements, and altered stances. Lim et al. used multi-modal SA to investigate balance improvements after a two-week balance training program involving 36 healthy older adults [50]. All participants wore a SA device $\left(\right.$ SwayStar $\left.{ }^{\mathrm{Tw}}\right)$, but only the experimental group received SA. Participants' trunk sway was monitored as they trained on the same seven standing and gait tasks for two consecutive weeks $(3 \times /$ week). Both experimental and control groups showed reduced body sway during the final training session, but training with SA provided little benefit over training alone. For most tasks, sway reductions did not persist in either group during immediate and onemonth post-training assessments. However, given that balance training is most effective following longer training periods (i.e., up to 12 weeks [16]), balance improvements and the persistence of the potential improvements between the groups may not have been realized given the relatively short training period.

To understand the efficacy of SA as a rehabilitation tool among community-dwelling older adults, this preliminary study investigated balance improvements after long-term (eight weeks) balance training with and without SA. We hypothesized that all participants would show improved clinical outcome scores after training, but that participants receiving SA would show greater improvements.

\section{Methods}

\section{Participants}

Twelve community-dwelling healthy older adults were recruited to participate following a screening session.
The sample size was partially informed by single day SA study findings $[23,33,51]$. Participants in the greater Ann Arbor, MI area were recruited via flyers and online advertisements on the website umhealthresearch.org. The recruiting period started in 2014 and ended in 2016. Participants were eligible for inclusion if they were 6585 years of age; medically stable; scored more than 26 points on the Montreal Cognitive Assessment; could stand unassisted for ten minutes; reported balance concerns $(\geq 1$ confirmative answer to balance perception questions, e.g., fear of falling, falls in the past year, losses of balance in the past 12 months, balance ratings $\geq 2$ on a five-point scale, Fig. 2); and could walk the distance of a city block without using an assistive device. Participants who had sustained a fall that required hospitalization or serious injury, had severe uncorrected vision or hearing loss, had a lower extremity fracture or sprain in the last six months or previous lower extremity joint replacement, had a history of a neurological condition (e.g., Parkinson's disease, multiple sclerosis, stroke), had motion-provoked vertigo or a diagnosed vestibular deficit, or had a body mass index larger than $30 \mathrm{~kg} / \mathrm{m}^{2}$ were excluded.

The twelve participants were randomly assigned to the experimental group (EG) or control group (CG) before pre-training assessments with a one-to-one allocation ratio. The EG ( $n=6,76.2 \pm 5.5$ yrs., 1 male/5 females) received vibrotactile SA during the training, while the CG completed the training without vibrotactile SA $(n=6$, $75.0 \pm 4.7$ yrs., 3 males $/ 3$ females). The study team randomized the participant assignments by blindly drawing sealed slips of paper with group designations. The first two participants were randomized in one block and the following ten participants were randomized in a second block. All participants gave written informed consent and the study was conducted in accordance with the Declaration of Helsinki. The study was reviewed and approved by the University of Michigan Institutional Review Board (HUM00086479).

\section{Protocol}

The experimental protocol, as shown in Fig. 1, comprised pre-training assessment with clinical balance testing (CBT), eight-week in-home balance training, mid-training assessment with CBT after a four-week training period, and post-training assessment with CBT. In-home balance training started within a week of the pre-training assessment and the post-assessment was completed within one week after training.

CBT, which included eight clinical outcome measures to evaluate balance and gait performance, was completed in the clinical setting by a physical therapist blinded to the participants' study group assignment (vibrotactile SA was not provided during $\mathrm{CBT}$ ): 


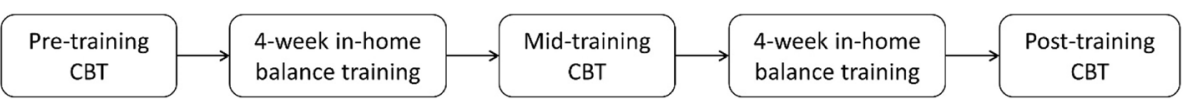

Fig. 1 Study protocol includes three clinical balance testing (CBT) sessions and eight weeks of in-home balance training

1) Activity-specific Balance Confidence ( $A B C$, out of 100) [52]: Identifies an individual's subjective measure of confidence in performing balance related activities of daily living. An ABC score of less than 67 indicates an increased risk for falling [53].

2) Computerized Dynamic Posturography: Sensory Organization Test (SOT) protocol [54]: Assesses an individual's ability to use their somatosensory, visual, and vestibular systems to maintain postural stability during standing, measured by the SOT composite score. Somatosensory, visual and vestibular reliance are calculated based on ratios of SOT scores to evaluate the reliance on each sensory system as shown below.

Somatosensory Reliance $=\frac{\text { SOT condition } 2}{\text { SOTcondition } 1}$

Visual Reliance $=\frac{\text { SOT condition } 4}{\text { SOT condition } 1}$

Vestibular Reliance $=\frac{\text { SOT condition } 5}{\text { SOT condition } 1}$

3) Mini Balance Evaluations Systems Test (Mini-BESTest) [55]: Uses 14 items to capture anticipatory postural adjustments, reactive postural control, sensory orientation, and dynamic gait performance. The Mini-BESTest was measured with two scoring systems: total score of 28 points (MiniBESTest28) uses the lower score of the left and right sides for unilateral balance tasks; total score of 32 points (MiniBESTest32) uses the cumulative score of both sides.

4) Five Times Sit to Stand Test (5xSST) [56]: Tests

functional lower limb muscle strength during transitional movements, measured in seconds. In older adults, a $5 x S S T$ duration equal to or greater than $12 \mathrm{~s}$ indicates a need for additional fall assessment [57].

5) Four Square Step Test (FSST) [58]: Assesses the ability to step over objects forward, sideways, and backwards, measured in seconds. FSST duration greater than $15 \mathrm{~s}$ for older adults indicates an increased risk for multiple falls [58].

6) Functional Reach Test (FRT) [59]: Assesses stability during maximum forward arm reach tasks while feet are in a fixed position, measured in centimeters. A FRT of less than $18 \mathrm{~cm}$ indicates limited mobility skills for older adults [60].

7) Ten-meter Walk Test: Assesses normal gait speed and fast gait speed, measured in meters per second. A substantial meaningful change in normal gait speed is $0.13 \mathrm{~m} / \mathrm{s}$ for older adults [61].

8) Timed Up and Go (TUG) and Timed Up and Go with Cognitive Task (TUG-COG) [62]: Assesses mobility, balance, and fall risk with and without a cognitive dual-task (count backwards by three), measured in seconds. For older adults, a TUG score greater than $13.5 \mathrm{~s}$ or a TUG-COG score greater than $15 \mathrm{~s}$ indicates fall risk [63].

After CBT but prior to beginning training, the treating physical therapist (different from the blinded assessor) and study team made one initial home visit to teach the participants how to use the smart phone balance trainer (detailed in the next section) and how to correctly perform independent in-home balance training exercises. Participants performed exercises from five categories as shown in Table 1. For exercises in Categories 1 (static standing) and 2 (compliant surface standing), participants performed static balance exercises on firm and foam surfaces, respectively. For Category 3 (weight shifting) exercises, participants were instructed to shift their body to and maintain their body at a target angle for five seconds in four directions (i.e., forward, backward, left and right). Movement angle was measured on the trunk, and the target angle was determined by the research team's physical therapists and was the same for all participants. For Category 4 (modified center of gravity)

Table 1 Exercise pool modified from a recently published conceptual progression framework [64]

\begin{tabular}{|c|c|}
\hline Category & Variables \\
\hline 1. Static standing ${ }^{a}$ & $\begin{array}{l}\text { Eyes, stance, head movement (yaw and pitch), } \\
\text { cognitive tasks }\end{array}$ \\
\hline $\begin{array}{l}\text { 2. Compliant surface } \\
\text { standing }^{\text {a }}\end{array}$ & Eyes, stance, head movement \\
\hline 3. Weight shifting ${ }^{a}$ & Shifting limit, shifting speed, shifting direction \\
\hline $\begin{array}{l}\text { 4. Modified center } \\
\text { of gravity }{ }^{a}\end{array}$ & $\begin{array}{l}\text { Arm raising speed, surface (firm, compliant } \\
\text { and ramps), head movement }\end{array}$ \\
\hline 5. Gait & $\begin{array}{l}\text { Walk with different speed and head movements, } \\
\text { high march }{ }^{a} \text {, step over shoe box }{ }^{a} \text {, sidestepping, } \\
\text { walk on heels/toes }{ }^{a} \text {, backward walking }{ }^{a} \text {, figure-of- } 8 \\
\text { walk, tandem }{ }^{a} \text {; cognitive tasks }\end{array}$ \\
\hline
\end{tabular}

andicates the exercises for which vibrotactile SA was provided for the EG 
exercises, participants repeatedly raised and lowered their arms from a resting position along the sides of their bodies with palms pronated and elbows locked, to $90^{\circ}$ of shoulder flexion. For Category 5 (gait) exercises, participants performed various overground locomotor tasks.

Participants were asked to exercise three times per week for eight weeks (24 sessions in total). For each session, participants were given a single exercise from each of the first four categories and two exercises from the fifth category; exercises were remotely recommended by the treating physical therapist. Each exercise was performed six times for $30 \mathrm{~s}$ (except Category 3 exercises where the trial stopped after participants maintained the target positions for five seconds). The training duration for each session was about $45 \mathrm{~min}$. Vibrotactile SA was provided to the EG via the smart phone balance trainer for all the exercises in the first four exercise categories and select exercises in the fifth category, as shown in Table 1. For these exercises, vibrotactile SA was provided during four randomly selected repetitions out of the six repetitions. The CG also wore the smart phone balance trainer, but never received vibrotactile SA. After each trial, participants were prompted by the smart phone to note any step-outs that occurred. A step-out was defined as taking a step to regain balance, touching a wall or chair for support, or opening one's eyes (on eyes closed tasks). After six repetitions participants rated their perceived stability on a visual analog scale (VAS) of 1-5 (see Fig. 2) [65]. The treating physical therapist used the reported number of step-outs and perceived stability scores to prescribe exercises weekly (three sessions per week) based on clinical experience and an exercise framework modified from a recently published conceptual progression framework [64]. The goal was to assign exercises that provided a moderate level of challenge, which was characterized by a score of 3 on the VAS. If there were no step outs and the participant rated the exercise a 1 on the VAS, a more challenging exercise was chosen; for example, adding pitch head movements would increase difficulty. If the exercises appeared too challenging (i.e., multiple step outs) and the participant rated the exercise a 4 or 5 on the VAS scale, an easier exercise was attempted until a moderately difficult exercise was found. The first set of exercises was determined during the initial home visit. Participants were asked to complete a weekly activity log to note pain that limited movement, falls, changes in medication, and any injuries from performing the exercises. MRIs were performed on a subset of the participants $(n=5)$ pre- and post-training for future analysis.

\section{Smart phone balance trainer}

A smart phone balance trainer was developed using design ethnography techniques during a co-creative design process involving engineers, physical therapists, and older adults [66]. The smart phone balance trainer comprised two Apple iPods (6th generation iPod touch, 2015), an elastic belt and a "tactor bud" accessory, as shown in Fig. 3. The two iPods are referred to as the "sensing" unit and "user interface" unit, respectively. The tactor bud contained a PCB-designed controller board, a 3.7 $\mathrm{V}$ battery, and four tactors (Precision Microdrives ${ }^{\mathrm{Tm}}$, 310-101 vibration motors encased in plastic housings [51]). The sensing unit was attached to an elastic belt and was worn around the torso at the L4/L5 level to measure trunk sway, and the user interface unit attached to a lanyard and was worn around the neck. The four tactors were aligned over the navel, lumbar spine, and right and left sides of the torso to provide directional vibrotactile cues.

Custom software (iOS application, Apple SDK) was developed to provide a semi-automated exercise progression routine with five exercise categories for inhome training, as shown in Fig. 4. Upon the launch of the software in the user interface unit, participants were asked to select an exercise to perform. Written, graphic and video instructions were presented on screen once the exercise was selected.

During each repetition, the sensing unit used gravitational outputs (Class CoreMotion, Apple Inc.) to estimate angular displacements (tilt angles) in the anterior-posterior and medial-lateral directions, adopted from Lee et al.'s algorithm [51]. Angular velocities were measured by the gyroscopes. Both accelerometers and gyroscopes were sampled at $50 \mathrm{~Hz}$. The user interface unit triggered the sensing unit to record trunk motion and the sensing unit informed the user interface unit of repetition completion via Bluetooth. The tactor

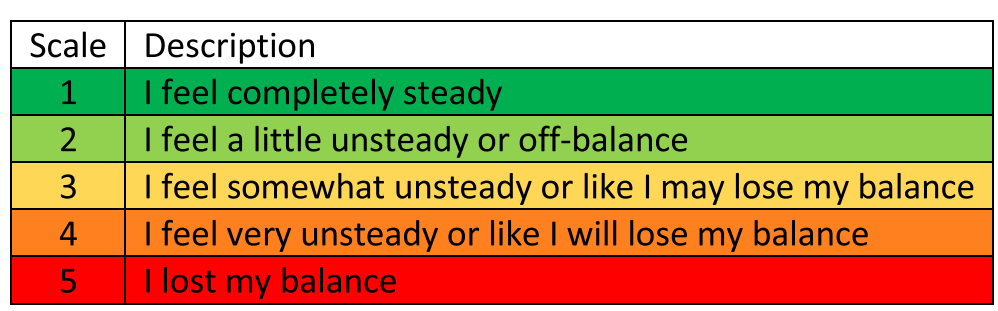

Fig. 2 Visual analog scale used by participants to rate their stability when performing the balance exercises 

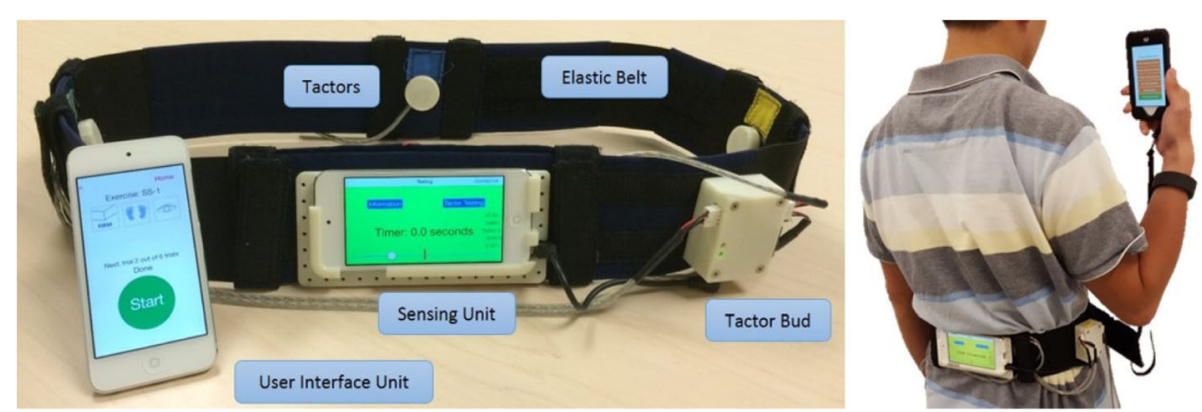

Fig. 3 Smart phone balance trainer

activation signal was defined as the tilt angle plus one half times the tilt angular rate for Categories 1, 2, 4 and 5 , and as the tilt angle for Category 3 exercises [24]. If the tactor activation signal exceeded a pre-set threshold [23, 38], the sensing unit sent audio output signals to the tactor bud accessory. The tactor bud accessory analyzed these audio signals and activated the corresponding tactor to provide vibrotactile cues. At the end of each repetition, the trunk motion data, number of step-outs, and visual analog scale ratings were automatically uploaded to a secured server via Wi-Fi.

\section{Statistical analysis}

Data are presented as group mean values plus or minus $( \pm)$ the standard deviation. Differences between the two groups at the pre-training assessment were tested using an independent samples two-tailed student's t-test. The effects of training with versus without SA on the clinical outcome measures were analyzed using a linear mixed model with group (experimental, control), time (pre-, mid-, post-training) and their interaction as fixed effects and the differences among individual participants as random effects. The measurements were logarithmically transformed if they were not normally distributed (e.g., 5xSST duration, fast gait speed, and TUG-COG duration). To investigate the time effects within each group, twotailed paired samples t-tests within each group were performed to detect statistically significant improvement, comparing mid- and post-training assessment with pretraining assessment. The significance level was set at 0.05 . Bonferroni corrections were used for the paired t-tests. Due to the relatively small sample size, the minimal detectable change (MDC) was also evaluated within groups. The MDC is defined as "a statistical estimate of the smallest amount of change that can be detected by a measure that corresponds to a noticeable change in ability" [67]. It reflects the minimal amount of change in a participant's score that ensures the change is not the result of measurement error, but is due to rehabilitation.

\section{Results}

There were no significant differences in age or gender between the EG and CG. All participants completed the training and the three CBTs without complaints, pains, falls, or injuries, which demonstrates the feasibility of the smart phone balance trainer for in-home balance training applications.

The data collected by the sensing unit indicated that without supervision from the study team, the EG participants were able to successfully use the vibrotactile SA in their homes to reduce their trunk sway. Fig. 5 shows illustrative data from Participant 6 performing an exercise (tandem Romberg stance on a firm surface with eyes open) in his home with and without vibrotactile SA provided by the smart phone balance trainer.

The two-tailed, independent samples t-test showed no significant differences for all clinical outcome measures between the two groups during the pre-training CBT $(p>0.1)$. Table 2 lists the results for a subset of the clinical outcome measures (SOT, MiniBESTest28, 5xSST) for all participants.

Table 3 shows the CBT assessment results for each group on average and the changes in clinical outcome measures from both pre-training to mid-training and pre-training to post-training. The linear mixed model showed significant main effects from pre-training assessments to post-training assessments in SOT composite score $(p<0.001)$, vestibular reliance $(p<0.01)$, Mini-BESTest28 $(\mathrm{p}<0.01)$ and Mini-BESTest32 $(p<0.01)$ and TUG-COG duration $(p<0.05)$. The linear mixed model also showed significant interaction effects between groups from pre-training assessments to post-training assessments in SOT composite score $(p<0.05)$, Mini-BESTest28 $(p<0.05)$, and Mini-BESTest32 $(p<0.05)$. These significant interaction effects indicate greater improvements for the EG than CG with average increases of 1.1, 0.40, and 0.58 points per week for the SOT composite scores, MiniBESTest28, and Mini-BESTest32, respectively. There were no significant interaction effects for the other CBT outcomes. The within-group paired t-test showed significant 


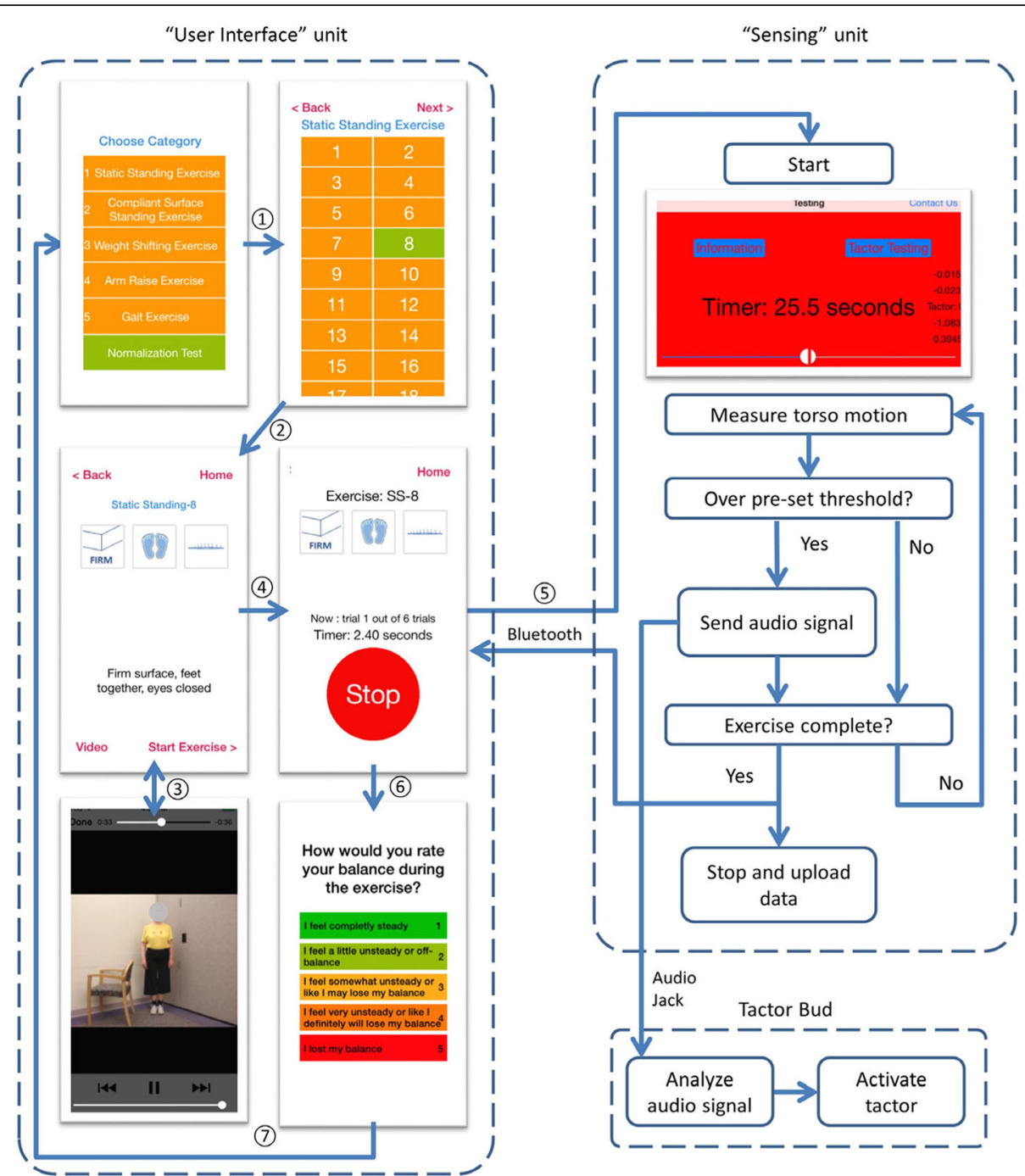

Fig. 4 Software schematics for the user interface and sensing units
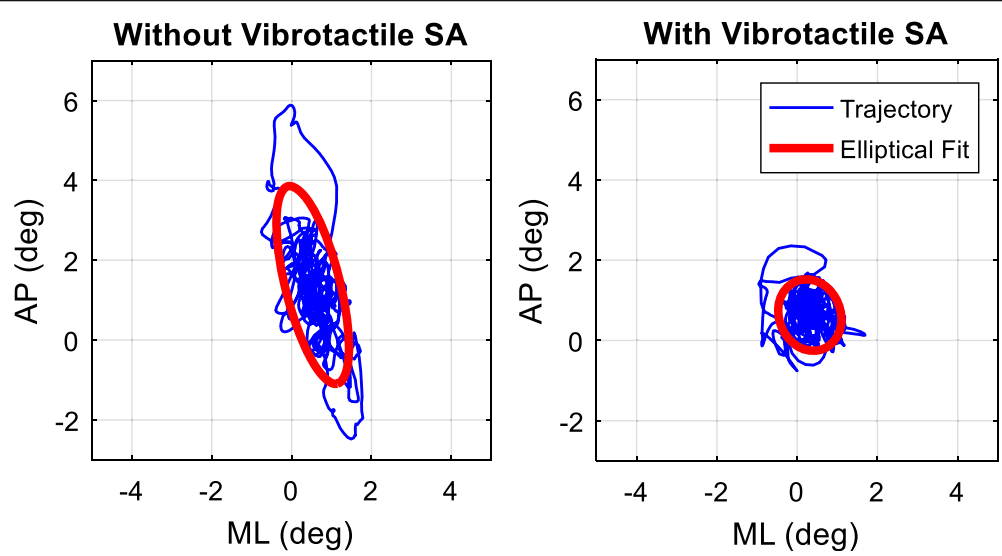

Fig. 5 Bird's-eye view of the body tilt trajectory in the anterior-posterior (AP) and medio-lateral (ML) directions for a sample exercise (tandem Romberg stance on firm surface with eyes open) performed by Participant 6 with and without vibrotactile SA 
Table 2 Participants' demographic information and results of a subset of clinical outcomes measures

\begin{tabular}{|c|c|c|c|c|c|c|c|c|c|c|c|c|}
\hline \multirow[t]{2}{*}{ Participant ID } & \multirow[t]{2}{*}{ Group } & \multirow[t]{2}{*}{ Age } & \multirow[t]{2}{*}{ Gender } & \multicolumn{3}{|l|}{ SOT } & \multicolumn{3}{|c|}{ Mini-BESTest28 } & \multicolumn{3}{|c|}{ 5xSST duration (s) } \\
\hline & & & & Pre & Mid & Post & Pre & Mid & Post & Pre & Mid & Post \\
\hline 1 & CG & 83 & M & 71 & 81 & 81 & 21 & 22 & 25 & 11.0 & 12.5 & 14.6 \\
\hline 2 & EG & 83 & $\mathrm{~F}$ & 63 & 72 & 83 & 22 & 24 & 25 & 12.0 & 10.3 & 11.0 \\
\hline 3 & EG & 70 & $F$ & 78 & 81 & 76 & 20 & 26 & 27 & 10.1 & 9.7 & 8.7 \\
\hline 4 & CG & 72 & $F$ & 49 & 45 & 46 & 25 & 25 & 24 & 7.4 & 9.3 & 11.0 \\
\hline 5 & $C G$ & 70 & M & 76 & 79 & 73 & 26 & 27 & 22 & 9.7 & 8.4 & 11.4 \\
\hline 6 & EG & 80 & M & 68 & 72 & 86 & 23 & 24 & 23 & 14.7 & 13.6 & 12.3 \\
\hline 7 & $C G$ & 73 & M & 60 & 68 & 65 & 24 & 25 & 25 & 8.8 & 10.0 & 8.3 \\
\hline 8 & EG & 70 & $\mathrm{~F}$ & 83 & 86 & 85 & 24 & 28 & 27 & 12.2 & 8.4 & 6.6 \\
\hline 9 & EG & 82 & $F$ & 74 & 78 & 79 & 25 & 25 & 26 & 17.8 & 14.2 & 15.0 \\
\hline 10 & $C G$ & 78 & $F$ & 83 & 84 & 85 & 23 & 22 & 25 & 13.7 & 9.7 & 9.9 \\
\hline 11 & CG & 74 & $F$ & 68 & 77 & 76 & 24 & 24 & 24 & 17.7 & 9.7 & 9.5 \\
\hline 12 & EG & 74 & $F$ & 50 & 73 & 79 & 19 & 26 & 26 & 14.6 & 11.1 & 10.6 \\
\hline
\end{tabular}

improvements for the EG in $5 \mathrm{xSST}$ duration during both mid- $(p<0.01)$ and post-training $(p<0.01)$. For the CG, there were no significant improvements on any of the CBT outcomes.

\section{Discussion}

This is the first study to investigate the effects of longterm (eight-week) balance training with and without vibrotactile SA on clinical outcome measures for community-dwelling older adults. Analysis of the twelve participants' scores showed that both the EG and CG had significant improvements in SOT composite scores, vestibular reliance, Mini-BESTest28, Mini-BESTest32 and TUG-COG duration; however, the EG improved significantly more than the CG in SOT composite scores, Mini-BESTest28, and Mini-BESTest32. In addition, significant improvements in $5 \mathrm{xSST}$ duration were found within the EG, whereas no significant improvements were found within the CG. However, no significant improvements were found in the $\mathrm{ABC}$ score,

Table 3 Clinical outcome measure results for pre-, mid- and post-training CBT and changes from pre-training CBT for the EG and CG. Average values with standard deviations are shown

\begin{tabular}{|c|c|c|c|c|c|c|c|c|c|c|}
\hline & \multicolumn{5}{|c|}{ Experimental Group } & \multicolumn{5}{|c|}{ Control Group } \\
\hline & Pre & Mid & Post & Mid - Pre & Post - Pre & Pre & Mid & Post & Mid - Pre & Post - Pre \\
\hline ABC score & $90.9 \pm 3.5$ & $89.4 \pm 6.4$ & $91.3 \pm 6.3$ & $-1.5 \pm 8.1$ & $0.5 \pm 8.7$ & $94.0 \pm 3.5$ & $91.8 \pm 4.2$ & $93.8 \pm 3.2$ & $-2.2 \pm 2.8$ & $-0.2 \pm 2.4$ \\
\hline SOT score $e^{a, b}$ & $69.3 \pm 11.8$ & $77.0 \pm 5.7$ & $81.3 \pm 3.9$ & $7.7 \pm 7.8$ & $12.0 \pm 12.1$ & $67.8 \pm 5.1$ & $72.3 \pm 12.0$ & $71.0 \pm 14.5$ & $4.5 \pm 5.5$ & $3.2 \pm 5.5$ \\
\hline Somatosensory reliance & $0.98 \pm 0.02$ & $0.98 \pm 0.02$ & $0.98 \pm 0.02$ & $0.00 \pm 0.03$ & $0.01 \pm 0.04$ & $0.95 \pm 0.02$ & $0.96 \pm 0.02$ & $0.95 \pm 0.02$ & $0.01 \pm 0.03$ & $0.00 \pm 0.02$ \\
\hline Visual reliance & $0.82 \pm 0.11$ & $0.86 \pm 0.07$ & $0.89 \pm 0.03$ & $0.04 \pm 0.11$ & $0.07 \pm 0.11$ & $0.87 \pm 0.05$ & $0.87 \pm 0.09$ & $0.88 \pm 0.10$ & $0.01 \pm 0.06$ & $0.01 \pm 0.08$ \\
\hline$V_{\text {Vestibular reliance }}{ }^{a}$ & $0.53 \pm 0.21$ & $0.63 \pm 0.20$ & $0.74 \pm 0.06$ & $0.10 \pm 0.08$ & $0.21 \pm 0.17$ & $0.40 \pm 0.28$ & $0.57 \pm 0.33$ & $0.56 \pm 0.31$ & $0.17 \pm 0.23$ & $0.16 \pm 0.24$ \\
\hline Mini-BESTest28 $8^{a, b}$ & $22.2 \pm 2.3$ & $25.5 \pm 1.5$ & $25.7 \pm 1.5$ & $3.3 \pm 2.8$ & $3.5 \pm 2.9$ & $23.8 \pm 1.7$ & $24.2 \pm 1.9$ & $24.2 \pm 1.2$ & $0.3 \pm 0.8$ & $0.3 \pm 2.7$ \\
\hline Mini-BESTest $32^{a, b}$ & $25.0 \pm 2.6$ & $28.7 \pm 2.2$ & $29.3 \pm 1.9$ & $3.7 \pm 3.5$ & $4.3 \pm 3.4$ & $26.8 \pm 2.0$ & $27.5 \pm 2.7$ & $26.5 \pm 1.4$ & $0.7 \pm 1.6$ & $-0.3 \pm 3.2$ \\
\hline 5xSST duration (s) & $13.5 \pm 2.7$ & $11.2 \pm 2.3$ & $10.7 \pm 2.9$ & $-2.4 \pm 1.4^{c}$ & $-2.9 \pm 1.7^{c}$ & $11.4 \pm 3.8$ & $9.9 \pm 1.4$ & $10.8 \pm 2.2$ & $-1.5 \pm 3.9$ & $-0.6 \pm 4.7$ \\
\hline FSST duration (s) & $9.9 \pm 2.2$ & $9.4 \pm 2.3$ & $9.5 \pm 2.1$ & $-0.6 \pm 0.6$ & $-0.6 \pm 0.5$ & $10.5 \pm 1.8$ & $10.8 \pm 1.5$ & $10.1 \pm 2.2$ & $0.3 \pm 1.5$ & $-0.4 \pm 1.2$ \\
\hline FRT (cm) & $35.0 \pm 6.1$ & $29.9 \pm 5.75$ & $31.9 \pm 5.0$ & $-5.1 \pm 4.3$ & $-3.0 \pm 4.2$ & $31.9 \pm 5.2$ & $28.9 \pm 3.3$ & $35.0 \pm 2.7$ & $-3.0 \pm 4.1$ & $3.1 \pm 3.8$ \\
\hline $\begin{array}{l}\text { Normal gait speed } \\
(\mathrm{m} / \mathrm{s})\end{array}$ & $1.22 \pm 0.08$ & $1.28 \pm 0.16$ & $1.27 \pm 0.19$ & $0.06 \pm 0.17$ & $0.06 \pm 0.20$ & $1.29 \pm 0.11$ & $1.23 \pm 0.14$ & $1.26 \pm 0.16$ & $-0.07 \pm 0.07$ & $-0.04 \pm 0.15$ \\
\hline Fast gait speed $(\mathrm{m} / \mathrm{s})$ & $1.62 \pm 0.24$ & $1.70 \pm 0.28$ & $1.69 \pm 0.29$ & $0.07 \pm 0.21$ & $0.06 \pm 0.31$ & $1.58 \pm 0.11$ & $1.57 \pm 0.18$ & $1.54 \pm 0.19$ & $-0.01 \pm 0.10$ & $-0.04 \pm 0.16$ \\
\hline TUG duration (s) & $10.8 \pm 2.1$ & $9.6 \pm 1.2$ & $10.5 \pm 1.4$ & $-1.1 \pm 1.8$ & $-0.3 \pm 1.4$ & $10.0 \pm 1.3$ & $9.8 \pm 1.6$ & $9.8 \pm 1.1$ & $-0.2 \pm 1.0$ & $-0.2 \pm 1.6$ \\
\hline TUG-COG duration $(s)^{a}$ & $13.3 \pm 2.9$ & $11.6 \pm 1.6$ & $11.8 \pm 2.1$ & $-1.7 \pm 1.8$ & $-1.5 \pm 1.4$ & $10.9 \pm 2.7$ & $11.0 \pm 2.3$ & $9.7 \pm 1.6$ & $0.2 \pm 1.8$ & $-1.1 \pm 2.8$ \\
\hline
\end{tabular}


somatosensory reliance, visual reliance, FSST duration, FRT, gait speed, and TUG duration.

After training, both groups showed improvements in the SOT composite score; significantly greater improvements were found for participants trained with SA than without SA (8 points vs. 5 points at mid-training, 12 points vs. 3 points at post-training on average). Prior studies have shown that when SA was provided, realtime sway reductions were noted for exercises in the SOT protocol [68]. The results of the current study indicate that after long-term training with SA, balance improvements in SOT protocol can be retained even when SA was not provided. SOT composite score improvements after training with SA (2-8 weeks) have been demonstrated in people with Parkinson's disease $(\sim 18$ points) [43], people with bilateral vestibular disorders ( 9 points) [45, 69], and people with other balance disorders ( $\sim 8$ points) [44]. Although the participants in our study reported no specific balance disorders, the EG exhibited similar improvements in SOT composite score ( $\sim 12$ points) after long-term training with SA. The MDC for the SOT composite score for young adults was previously determined to be greater than 8.1 points [70]. In this study, three participants in the EG achieved MDCs in SOT composite scores, while only one participant in the CG achieved a MDC. Furthermore, these three EG participants improved by at least 15 points, while the CG participant improved by 10 points. These results indicate that training with SA may be more effective than training alone for achieving MDCs in SOT performance. From mid-training to post-training, the EG showed continuous improvements, while the CG showed a plateau effect, which suggests that training with SA could result in higher potential improvement than training without SA.

Somatosensory, visual, and vestibular reliance were calculated using SOT Conditions 1, 2, 4, and 5. Somatosensory reliance did not significantly improve following training, however, the margin for improvement was limited by high levels of somatosensory reliance prior to training. Participants in both the EG and CG relied more on visual and vestibular inputs for maintaining balance after training, although vestibular reliance showed a larger increase. These shifts in visual and vestibular reliance may support the "reweighting" hypothesis for balance training [41]. Increased vestibular reliance may be attributed to performing exercises with eyes closed and/ or incorporating head movements [71]. Moreover, greater increases on vestibular reliance were observed in the EG, which may suggest that training with SA has a greater impact on reweighting vestibular inputs than training without SA.

The EG showed significantly greater improvements than the CG for both Mini-BESTest28 and Mini-
BESTest32 scores. Training with SA while performing static and dynamic standing and gait exercises could explain this difference because the Mini-BESTest assesses dynamic balance [55]. Although no MDC data are available for older adults, Godi et al. reported a 3.5-point MDC for the Mini-BESTest28 among people with Parkinson's disease (baseline Mini-BESTest28 value was 12.8 points) [72]. In our study, three participants in the EG demonstrated a 3.5-point change during midtraining CBT versus no participants in the CG. The average baseline Mini-BESTest28 value for all participants was 22.2 points.

Within-group analysis of 5xSST performance showed significant improvement of test duration for the EG but not for the CG after training concluded. Additionally, all participants in the EG improved their 5xSST durations after training, but only three out of the six participants in the CG showed improvements. Given that improvements in $5 \mathrm{xSST}$ duration are correlated with improved lower limb muscle strength and stability during transitional movements [56], training with SA may be more effective for improving functional mobility. It was also noted that two participants from each group reduced their 5xSST durations to less than $12 \mathrm{~s}$ (a fall risk indicator [57]) at the mid- and post-training CBTs. Finally, although the sit-tostand task was not an exercise performed during balance training, training effects from dynamic standing tasks (especially Category 3, Weight Shifting exercises, and Category 4, Modified Center of Gravity exercises) may have been transferred to the sit-to-stand task.

No significant changes in $A B C$ scores were found in either the EG or the CG, although scores generally declined from pre-training to mid-training and increased from mid-training to post-training. As healthy older adults, all participants had relatively high $\mathrm{ABC}$ scores $(>85)$ at the pre-training assessment, and were therefore unlikely to show further improvement in balance confidence (i.e., ceiling effect) [73]. Declines in scores from pre- to mid-training are consistent with an initial overconfidence in balance abilities and a shift in awareness of limitations [74]. Score increases from mid- to post-training may reflect improvements in overall balance performance, although the improvements were relatively small.

The EG and CG showed significant improvements in TUG-COG duration, possibly due to the effects of training with cognitive tasks. However, no significant improvements for FRT distance, FSST duration, normal gait speed, fast gait speed, and TUG duration were noted. The lack of significant improvements may have been due to ceiling effects, or the difficulty level of the selected exercises may have been below the necessary level to elicit improvements for these outcomes. However, on average, the EG showed greater 
improvements in normal gait speed and fast gait speed than the CG. Additionally, one participant in the EG achieved a MDC for normal gait speed $(0.18 \mathrm{~m} / \mathrm{s}$ for Parkinson's Disease) and two participants in the EG achieved a MDC for fast gait speed $(0.25 \mathrm{~m} / \mathrm{s}$ for Parkinson's Disease) [75]. No participants achieved MDCs in the CG. Transfer effects from using SA during static and dynamic exercises may account for observed differences.

Our findings appear to contradict those of Lim et al., who found no significant differences in body sway between a group training with $\mathrm{SA}$ and a group training without SA after a two-week program [50]; however, these two studies have several important differences. First, participants in Lim et al.'s study trained for two weeks $(3 \times /$ week $)$ with six training sessions in total, whereas in this study all participants trained for eight weeks $(3 \times /$ week) with 24 training sessions in total. As shown by Lesinski et al., longer training periods result in greater improvements in balance performance [16]. Second, Lim et al.'s study provided SA to the experimental group during all exercise repetitions during all sessions (i.e., 100\% feedback), whereas this study provided SA to the EG for four out of the six repetitions for each exercise (i.e., $67 \%$ feedback). Feedback can have negative effects if provided too frequently. Winstein and Schmidt found that providing feedback for the entire duration of motor skill training can improve short term performance but limit motor learning, while providing feedback for only portions of training produces poor initial performance results but improves motor skill retention [76, 77]. Therefore, training with reduced feedback frequency in this study may have improved skill retention after training concluded. Third, Lim et al. trained all participants using the same exercises throughout all sessions regardless of the participants' balance abilities, whereas in this study a physical therapist selected the exercises performed by each participant based on his/her historical performance. Training with a constant set of exercises may limit the margin for improvement among high functioning participants, while those with poorer balance ability may experience larger improvements. In balance rehabilitation programs, experienced physical therapists progress balance exercises to achieve greater balance improvements [64]. Individualized exercise selection in this study allows participants to perform progressively challenging exercises throughout the entire training program to maximize improvement regardless of skill level. Fourth, Lim et al. quantified the effects of balance training by comparing measures of trunk motion, whereas this study used clinical outcome measures to evaluate improvements.

This study employed some elements of telerehabilitation to monitor performance and provide custom exercise regimens. Body motion, subjective ratings of balance and number of step outs were captured on the smart phone balance trainer during home-based balance training. This information was sent to a physical therapist via a wireless internet connection. Exercise programs were customized based on performance data and updated regimens were sent from the physical therapist to the participants via email. Conceptually, this aligns with telerehabilitation models, which deliver remote rehabilitation services, including assessments and interventions, via telecommunication networks [78]. However, our paradigm required less expert (i.e., physical therapist) engagement with participants as compared to traditional programs because we provided within-session vibrotactile SA as instructional balance cues. Physical therapist's time commitments were limited to less than thirty minutes per week per participant and focused on analysis of previous balance performance and customization of the rehabilitation program, whereas typical telerehabilitation programs generally engage the expert and user remotely for the duration of the training session [79]. The findings of this study are consistent with prior work that has shown improved balance performance following telerehabilitation interventions for both people with balance deficits and community dwelling older adults [79-83]. Previously published research has also demonstrated potential economic benefits to using telerehabilitation approaches $[79,80]$. While cost effectiveness was not explored as part of this study, the smart phone balance trainer $(<\$ 1 \mathrm{k})$ coupled with reduced patient-expert interaction could reduce the overall cost of providing rehabilitative care for a subset of people with balance deficits and simultaneously mitigate future costs stemming from injurious loss-of-balance events. Overall, improvements in clinical outcomes support the potential use of a smart phone balance trainer as a telerehabilitation tool.

Our study is not without limitations. First, vibrotactile SA was only provided during a subset of exercises under the gait category because few studies have addressed the effectiveness of SA for improving stability during locomotor tasks [22]. Typical feedback strategies during gait activities include walking in step with auditory or visual cues or vibrotactile cues presented to a single body segment or joint to warn of extension beyond a desired angle [32, 38, 84]. Sienko et al. provided continuous vibrotactile SA based on trunk motion during overground locomotion, but slightly reduced trunk sway was observed in a subset of the trials and some participants demonstrated stiffening in the coronal plane [38]. Second, although this study employed an experienced physical therapist to instruct participants on correct exercise performance, provided handouts with instructional text and pictures, and provided exercise videos, correctness of exercise performance was not monitored during 
training because training occurred in participants' homes. Third, despite the statistical and clinical significances found in this study, the sample size was relatively small. Finally, although this study used an experienced physical therapist to recommend the exercises remotely, the information provided to the physical therapist by the smart phone balance trainer was limited to the number of step-outs in the six repetitions and the stability perception ratings from the participants. A more sophisticated algorithm that captures exercise performance more comprehensively could help therapists make better recommendations in the future.

\section{Conclusions}

In-home balance training with vibrotactile SA for eight weeks improved balance performance of communitydwelling healthy older adults in this preliminary study. Participants trained with SA improved more than those trained without SA, particularly in SOT composite, Mini-BESTest, and 5xSST performance. Balance training with SA also increased visual and vestibular reliance, and improved static and dynamic balance, compared to training without SA. The lack of significant improvements in gait-related clinical outcome measures may be due to the lack of meaningful SA provided when performing gait exercises and the limited transfer effects from performance of standing exercises. All participants completed the eight-week training protocol with no reports of pain, injuries, or falls, which suggests that healthy older adults are able to use the smart phone balance trainer safely and independently. Overall, this study supports SA as a balance rehabilitation tool and potential telerehabilitation tool for use by communitydwelling healthy older adults.

\section{Abbreviations \\ 5xSST: Five Times Sit to Stand Test; ABC: Activity-specific Balance Confidence; CBT: Clinical Balance Test; CG: Control Group; EG: Experimental Group; FRT: Functional Reach Test; FSST: Four Square Step Test; MDC: Minimal Detectable Change; Mini-BESTest: Mini Balance Evaluations Systems Test; SA: Sensory Augmentation; SOT: Sensory Organization Test; TUG: Timed Up and Go; TUG-COG: Timed Up and Go with Cognitive Task; VAS: Visual Analog Scale}

\section{Acknowledgments}

We thank Jaehong Lee for his assistance with device development, and Xingjian Lai and Lydia Su for their assistance with data collection.

\section{Funding}

This work was supported by NSF (GARDE 1159635 and CAREER RAPD/GARDE0846471), NIH (5R21DC012410-02) and the University of Michigan Pepper Center (NIH RCDC/KL2 2P30AG024824-11).

\section{Availability of data and materials}

The data collected and/or analyzed in this study are available from the corresponding author on reasonable request.

\section{Authors' contributions}

$T B, V B$, and $K S$ contributed to the development of the device. TB, WC, CK, SW, and KS contributed to the experimental design. WC, CK, GP, SW, and KS contributed to the development of the balance training exercise program, exercise selection and progression, exercise instructions. TB, WC, CK, VB and GP collected the data. TB, WC, MK, RS, and KS contributed to data analysis. All authors contributed to the preparation of the manuscript and the approved the final version. KS supervised the research.

\section{Ethics approval and consent to participate}

All participants gave written informed consent and the study was conducted in accordance with the Declaration of Helsinki. The study was reviewed and approved by the University of Michigan Institutional Review Board (HUM00086479).

\section{Consent for publication}

All participants gave written informed consent.

\section{Competing interests}

The authors declare that they have no competing interests.

\section{Publisher's Note}

Springer Nature remains neutral with regard to jurisdictional claims in published maps and institutional affiliations.

\section{Author details}

'Department of Mechanical Engineering, University of Michigan, 2350 Hayward St., Ann Arbor 48109, MI, USA. ${ }^{2}$ Department of Otolaryngology, Michigan Medicine, University of Michigan, 1500 E Medical Center Dr, Ann Arbor, MI 48109, USA. ${ }^{3}$ Physical Medicine \& Rehabilitation, Michigan Medicine, University of Michigan, 1500 E Medical Center Dr, Ann Arbor, Ml 48109, USA. ${ }^{4}$ Department of Physical Therapy and Otolaryngology, School of Health and Rehabilitation Sciences, University of Pittsburgh, 4028 Forbes Tower, Pittsburgh, PA 15260, USA. ${ }^{5}$ Department of Internal Medicine, University of Michigan, 1500 East Medical Center Drive, Ann Arbor 48109, MI, USA. ${ }^{6}$ School of Kinesiology, University of Michigan, 1402 Washington Heights, Ann Arbor, Ml 48109, USA. ${ }^{7}$ Department of Psychology, University of Michigan, 530 Church St, Ann Arbor, MI 48109, USA. ${ }^{8}$ Department of Applied Physiology and Kinesiology, University of Florida, Gainesville, USA.

${ }^{9}$ Department of Biomedical Engineering, University of Michigan, 2200 Bonisteel Blvd, Ann Arbor, Ml 48109, USA.

Received: 13 June 2017 Accepted: 11 December 2017

Published online: 18 January 2018

\section{References}

1. Maki BE, Mcllroy WE. Postural control in the older adult. Clin Geriatr Med. 1996;12:635-58.

2. Benjuya N, Melzer I, Kaplanski J. Aging-induced shifts from a reliance on sensory input to muscle cocontraction during balanced standing. J Gerontol Med Sci. 2004;59A:166-71.

3. Shaffer SW, Harrison AL. Aging of the somatosensory system: a translational perspective. Phys Ther. 2007:87:193-207

4. Delbaere K, Close JCT, Heim J, Sachdev PS, Brodaty H, Slavin MJ, Kochan NA Lord SR. A multifactorial approach to understanding fall risk in older people. J Am Geriatr Soc. 2010;58:1679-85.

5. Horak FB. Postural orientation and equilibrium: what do we need to know about neural control of balance to prevent falls? Age Ageing. 2006; 35(SUPPL.2):7-11.

6. Tinetti ME. Preventing falls in elderly persons. N Engl J Med. 2003;348:42-9.

7. Scheffer AC, Schuurmans MJ, van Dijk N, van der Hooft T, de Rooij SE. Fear of falling: measurement strategy, prevalence, risk factors and consequences among older persons. Age Ageing. 2008;37:19-24.

8. Donoghue OA, Cronin H, Savva GM, O'Regan C, Kenny RA. Effects of fear of falling and activity restriction on normal and dual task walking in community dwelling older adults. Gait Posture. 2013;38:120-4.

9. Rejeski WJ, Mihalko SL. Physical activity and quality of life in older adults. J Gerontol Ser A Biol Sci Med Sci. 2001;56(Supplement 2):23-35.

10. Shumway-Cook A, Gruber W, Baldwin M, Liao S. The effect of multidimensional exercises on balance, mobility, and fall risk in communitydwelling older adults. Phys Ther. 1997;77:46-57.

11. $\mathrm{MH} \mathrm{H}$, Woollacott $\mathrm{MH}$. Multisensory training of standing balance in older adults: I. Postural stability and one-leg stance balance. J Gerontol. 1994;49:M52-61.

12. Herdman SJ, Blatt $P$, Schubert MC, Tusa RJ. Falls in patients with vestibular deficits. Am J Otol. 2000;21:847-51. 
13. Tinetti ME, Williams CS. The effect of falls and fall injuries on functioning in community-dwelling older persons. J Gerontol Ser A Biol Sci Med Sci. 1998; 53A:M112-9.

14. Hauer K, Rost B, Rütschle K, Hedda O, Specht N, Bärtsch P, Oster P, Schlierf G. Exercise Training for Rehabilitation and Secondary Prevention of Falls in Geriatric Patients With A History of Injurous Falls. J Am Geriatr Soc. 2001;49: 10-20.

15. Gillespie LD, Robertson MC, Gillespie WJ, Sherrington C, Gates S, Clemson LM, Lamb SE. Interventions for preventing falls in older people living in the community. In: Gillespie LD, editor. Cochrane database of systematic reviews. John Wiley \& Sons, Ltd: Chichester, UK; 2012

16. Lesinski M, Hortobágyi T, Muehlbauer T, Gollhofer A, Granacher U. Effects of balance training on balance performance in healthy older adults: a systematic review and meta-analysis. Sport Med. 2015;45:1721-38.

17. Kao CL, Chen LK, Chern CM, Hsu LC, Chen CC, Hwang SJ. Rehabilitation outcome in home-based versus supervised exercise programs for chronically dizzy patients. Arch Gerontol Geriatr. 2009;51:264-7.

18. Lacroix A, Kressig RW, Muehlbauer T, Gschwind YJ, Pfenninger B, Bruegger O, Granacher U. Effects of a Supervised versus an Unsupervised Combined Balance and Strength Training Program on Balance and Muscle Power in Healthy Older Adults: A Randomized Controlled Trial. Gerontology. 2015;62: 275-88

19. Bach-y-Rita P, W Kercel S. Sensory substitution and the human-machine interface. Trends Cogn Sci. 2003;7:541-6.

20. Wall C, Weinberg MS. Balance prostheses for postural control. IEEE Eng Med Biol Mag. 2003;22:84-90.

21. Jeka JJ. Light touch contact as a balance aid. Phys Ther. 1997;77:476-87.

22. Sienko KH, Whitney SL, Carender WJ, Wall C III. The role of sensory augmentation for people with vestibular deficits: real-time balance aid and/ or rehabilitation device? J Vestib Res. 2017;27:63-76.

23. Sienko KH, Balkwill MD, Oddsson LIE, Wall C. Effects of multi-directional vibrotactile feedback on vestibular-deficient postural performance during continuous multi-directional support surface perturbations. J Vestib Res. 2008;18:273-85.

24. Wall C III, Kentala E. Effect of displacement, velocity, and combined vibrotactile tilt feedback on postural control of vestibulopathic subjects. J Vestib Res. 2010;20:61-9.

25. Sienko KH, Balkwill MD, Wall C. Biofeedback improves postural control recovery from multi-axis discrete perturbations. J Neuroeng Rehabil. 2012;9:53.

26. Bechly KE, Carender WJ, Myles JD, Sienko KH. Determining the preferred modality for real-time biofeedback during balance training. Gait Posture. 2013;37:391-6

27. Wall C, Wrisley DM, Statler KD. Vibrotactile tilt feedback improves dynamic gait index: a fall risk indicator in older adults. Gait Posture. 2009;30:16-21.

28. Janssen M, Stokroos R, Aarts J, van Lummel R, Kingma H. Salient and placebo vibrotactile feedback are equally effective in reducing sway in bilateral vestibular loss patients. Gait Posture. 2010;31:213-7.

29. Allum JHJ, Carpenter MG, Horslen BC, Davis JR, Honegger F, Tang K-S, Kessler P. Improving impaired balance function: real-time versus carry-over effects of prosthetic feedback. Conf Proc IEEE Eng Med Biol Soc. 2011;2011:1314-8.

30. Halická Z, Lobotková J, Bučková K, Hlavačka F. Effectiveness of different visual biofeedback signals for human balance improvement. Gait Posture. 2014;39:410-4.

31. Dozza M, Horak FB, Chiari L. Auditory biofeedback substitutes for loss of sensory information in maintaining stance. Exp Brain Res. 2007;178:37-48.

32. Hegeman J, Honegger $F$, Kupper M, Allum JHJ. The balance control of bilateral peripheral vestibular loss subjects and its improvement with auditory prosthetic feedback. J Vestib Res. 2005;15:109-17.

33. Davis JR, Carpenter MG, Tschanz R, Meyes S, Debrunner D, Burger J, Allum JHJ. Trunk sway reductions in young and older adults using multi-modal biofeedback. Gait Posture. 2010;31:465-72.

34. Bach-y-Rita P, Danilov Y, Tyler M, Grimm RJ. Late human brain plasticity: vestibular substitution with a tongue BrainPort human-machine interface. Intellectica. 2005;1:115-22.

35. Goodworth AD, Wall C, Peterka RJ. Influence of feedback parameters on performance of a vibrotactile balance prosthesis. IEEE Trans Neural Syst Rehabil Eng. 2009;17:397-408.

36. Goodworth AD, Wall C, Peterka RJ. A balance control model predicts how vestibular loss subjects benefit from a vibrotactile balance prosthesis. In: Annual international conference of the IEEE EMBS; 2011. p. 1306-9.

37. Bach-y-Rita P. Tactile sensory substitution studies. Ann N Y Acad Sci. 2004; 1013:83-91.
38. Sienko KH, Balkwill MD, Oddsson LIE, Wall C. The effect of vibrotactile feedback on postural sway during locomotor activities. J Neuroeng Rehabil. 2013;10:93.

39. Dozza M, Wall C, Peterka RJ, Chiari L, Horak FB. Effects of practicing tandem gait with and without vibrotactile biofeedback in subjects with unilateral vestibular loss. J Vestib Res. 2007:17:195-204.

40. Janssen LJF, Verhoeff LL, Horlings CGC, Allum JHJ. Directional effects of biofeedback on trunk sway during gait tasks in healthy young subjects. Gait Posture. 2009;29:575-81.

41. Horak FB. Postural compensation for vestibular loss and implications for rehabilitation. Restor Neurol Neurosci. 2010;28:57-68.

42. Danilov YP, Tyler ME, Skinner KL, Bach-y-Rita P. Efficacy of electrotactile vestibular substitution in patients with bilateral vestibular and central balance loss. In: IEEE EMBS annual international conference; 2006. p. 6605-9.

43. Rossi-Izquierdo M, Ernst A, Soto-Varela A, Santos-Pérez S, Faraldo-García A, Sesar-Ignacio A, Basta D. Vibrotactile neurofeedback balance training in patients with Parkinson's disease: reducing the number of falls. Gait Posture. 2013;37:195-200.

44. Basta D, Rossi-Izquierdo M, Soto-Varela A, Greters ME, Bittar RS, SteinhagenThiessen E, Eckardt R, Harada T, Goto F, Ogawa K, Ernst A. Efficacy of a vibrotactile neurofeedback training in stance and gait conditions for the treatment of balance deficits: a double-blind, placebo-controlled multicenter study. Otol Neurotol. 2011;32:1492-9.

45. Brugnera C, Bittar RSM, Greters ME, Basta D. Effects of vibrotactile vestibular substitution on vestibular rehabilitation - preliminary study. Braz J Otorhinolaryngol. 2015;81:616-21.

46. Jorgensen MG, Laessoe U, Hendriksen C, Nielsen OBF, Aagaard P. Efficacy of nintendo wii training on mechanical leg muscle function and postural balance in community-dwelling older adults: a randomized controlled trial. Journals Gerontol - Ser A Biol Sci Med Sci. 2013;68:845-52.

47. Whyatt C, Merriman NA, Young WR, Newell FN, Craig C. A Wii bit of fun: a novel platform to deliver effective balance training to older adults. Games Health J. 2015:4:1-11.

48. Roopchand-Martin S, McLean R, Gordon C, Nelson G. Balance training with Wii fit plus for community-dwelling persons 60 years and older. Games Health J. 2015:4:247-52.

49. AS F, Gao KL, Tung AK, Tsang WW, Kwan MM. Effectiveness of Exergaming training in reducing risk and incidence of falls in frail older adults with a history of falls. Arch Phys Med Rehabil. 2015;96:2096-102.

50. Lim SB, Horslen BC, Davis JR, Allum JHJ, Carpenter MG. Benefits of multisession balance and gait training with multi-modal biofeedback in healthy older adults. Gait Posture. 2016;47:10-7.

51. Lee B-C, Kim J, Chen S, Sienko KH. Cell phone based balance trainer. J Neuroeng Rehabil. 2012;9:10

52. Powell LE, Myers AM. The activities-specific balance confidence (ABC) scale. Journals Gerontol Ser A Biol Sci Med Sci. 1995;50:M28-34.

53. Lajoie Y, Gallagher SP. Predicting falls within the elderly community: comparison of postural sway, reaction time, the berg balance scale and the activities-specific balance confidence (ABC) scale for comparing fallers and non-fallers. Arch Gerontol Geriatr. 2004;38:11-26.

54. Clendaniel RA. Outcome measures for assessment of treatment of the dizzy and balance disorder patient. Otolaryngol Clin N Am. 2000;33:519-33.

55. Franchignoni F, Horak F, Godi M, Nardone A, Giordano A. Using psychometric techniques to improve the balance evaluation systems test: the mini-bestest. J Rehabil Med. 2010;42:323-31.

56. Csuka M, McCarty DJ. Simple method for measurement of lower extremity muscle strength. Am J Med. 1985;78:77-81.

57. Tiedemann A, Shimada H, Sherrington C, Murray S, Lord S. The comparative ability of eight functional mobility tests for predicting falls in communitydwelling older people. Age Ageing. 2008;37:430-5.

58. Dite W, Temple VA. A clinical test of stepping and change of direction to identify multiple falling older adults. Arch Phys Med Rehabil. 2002;83:1566-71.

59. Duncan PW, Weiner DK, Chandler J, Studenski S. Functional reach: a new clinical measure of balance. J Gerontol. 1990;45:M192-7.

60. Weiner DK, Duncan PW, Chandler J, Studenski S. A: functional reach: a marker of physical frailty. J Am Geriatr Soc. 1992:40:203-7.

61. Perera S, Mody SH, Woodman RC, Studenski SA. Meaningful change and responsiveness in common physical performance measures in older adults. J Am Geriatr Soc. 2006;54:743-9.

62. Podsiadlo D, Richardson S. The timed "up \& go": a test of basic functional mobility for frail elderly persons. J Am Geriatr Soc. 1991;39:142-8. 
63. Shumey-Cook A, Brauer S, Woollacott M. Predicting the probability for falls in community-dwelling older adults using the Timed Up \& Go Test. Phys Ther. 2000;80:896-03.

64. Klatt BN, Carender WJ, Lin C, Alsubaie S, Kinnaird CR, Sienko KH, Whitney SL. A Conceptual Framework for the Progression of Balance Exercises in Persons with Balance and Vestibular Disorders. Phys Med Rehabil - Int. 2015; 2:1044.

65. Espy D, Nathan C, Tony W, KMR N. Development of a rating scale for perceived stability during balance training. In: International Society for Posture and Gait Research; 2014.

66. Carender W, Kinnaird C, Peethambaran G, Fantich G, Whitney S, Sienko K. Balance telerehabilitation: considering user preferences for the design of a cell phone balance trainer. In: International Society for Posture and Gait Research; 2015.

67. Rehabilitation Measures Database [http://www.rehabmeasures.org/ rehabweb/rhstats.aspx].

68. Wall C III, Kentala E. Control of sway using vibrotactile feedback of body tilt in patients with moderate and severe postural control deficits. J Vestib Res. 2005;15:313-25.

69. Bao T, Klatt B, Carender W, Kinnaird C, Whitney S, Sienko K. Long-term balance training with vibrotactile biofeedback: two case reports. In: International Society for Posture and Gait Research; 2015.

70. Wrisley DM, Stephens MJ, Mosley S, Wojnowski A, Duffy J, Burkard R. Learning effects of repetitive administrations of the sensory organization test in healthy young adults. Arch Phys Med Rehabil. 2007;88:1049-54.

71. Cenciarini M, Peterka RJ, Cenciarini M, Peterka RJ. Stimulus-Dependent Changes in the Vestibular Contribution to Human Postural Control StimulusDependent Changes in the Vestibular Contribution to Human Postural Control. J Neurophysiol. 2006;95:2733-750.

72. Godi M, Franchignoni F, Caligari M, Giordano A, Turcato AM, Nardone A. Comparison of Reliability, Validity, and Responsiveness of the Mini- BESTest and Berg Balance Scale in Patients With Balance Disorders. Phys Ther. 2013; 93:158-67.

73. Myers AM, Fletcher PC, Myers AH, Sherk W. Discriminative and evaluative properties of the activities-specific balance confidence (ABC) scale. J Gerontol Med Sci. 1998;53:287-94.

74. Fortinsky RH, Panzer V, Wakefield D, Into F. Alignment between balance confidence and fall risk in later life: has over-confidence been overlooked? Health Risk Soc. 2009;11:341-52.

75. Steffen T, Seney M. Form health survey, and the unified Parkinson disease rating scale in people with parkinsonism. Phys Ther. 2008;88:733-46.

76. Winstein CJ, Schmidt RA. Reduced frequency of knowledge of results enhances motor skill learning. J Exp Psychol Learn Mem Cogn. 1990;16:677-91.

77. Schmidt RA: Frequent augmented feedback can degrade learning: Evidence and interpretations. In Tutorials in motor neuroscience. Dordrecht, The Netherlands Springer. 1991;59-75.

78. Burns RB, Crislip D, Daviou P, Temkin A, Vesmarovich S, Anshutz J, Furbish C, Jones ML. Using telerehabilitation to support assistive technology. Assist Technol. 1998;10:126-33.

79. Kairy D, Lehoux P, Vincent C, Visintin M. A systematic review of clinical outcomes, clinical process, healthcare utilization and costs associated with telerehabilitation. Disabil Rehabil. 2009;31:427-47.

80. Tousignant M, Boissy P, Corriveau H, Moffet $\mathrm{H}$. In home telerehabilitation for older adults after discharge from an acute hospital or rehabilitation unit: a proof-of-concept study and costs estimation. Disabil Rehabil Assist Technol. 2006; 1:209-16.

81. Lai JCK, Woo J, Hui E, Chan WM. Community-based stroke rehabilitation: 2004. p. 199-205.

82. Llorens R, Noe E, Colomer C, Alcaniz M. Effectiveness, usability, and costbenefit of a virtual reality-based telerehabilitation program for balance recovery after stroke: a randomized controlled trial. Arch Phys Med Rehabil. 2015;96:418-425.e2.

83. Cikajlo I, Rudolf M, Goljar N, Burger H, Matjačić Z. Telerehabilitation using virtual reality task can improve balance in patients with stroke. Disabil Rehabil. 2012;34:13-8.

84. Shull PB, Lurie KL, Cutkosky MR, Besier TF. Training multi-parameter gaits to reduce the knee adduction moment with data-driven models and haptic feedback. J Biomech. 2011:44:1605-9.

\section{Submit your next manuscript to BioMed Central and we will help you at every step:}

- We accept pre-submission inquiries

- Our selector tool helps you to find the most relevant journal

- We provide round the clock customer support

- Convenient online submission

- Thorough peer review

- Inclusion in PubMed and all major indexing services

- Maximum visibility for your research

Submit your manuscript at www.biomedcentral.com/submit
Biomed Central 